\title{
Relação entre tempo de transplante renal e força muscular respiratória: série de casos
}

\author{
Relationship between renal transplantation time and respiratory \\ muscle strength: case series
}

\section{Carla Cristina Braga Maranhão' \\ Lívia Gomes da Rocha ${ }^{2}$ \\ Helga Cecília Muniz de Souza ${ }^{3}$ \\ Patrícia Érika de Melo Marinho4 \\ Endereço para correspondência: \\ Patrícia Érika M Marinho \\ Departamento de Fisioterapia, Universidade Federal de Pernambuco \\ Av. Jornalista Aníbal Fernandes, s/n - Cidade Universitária 50740-560 - Recife, PE [Brasil] \\ patricia.marinho@ufpe.br; patmarinho@yahoo.com.br}

\footnotetext{
1 Programa de Residência Multiprofissional Integrada em Saúde do Hospital das Clínicas da Universidade Federal de Pernambuco - UFPE. Recife, PE, Brasil. ORCID: https://orcid.org/0000-0003-4129-938X

2 Programa de Pós-Graduação em Fisioterapia da Universidade Federal de Pernambuco - UFPE. Recife, PE - Brasil. ORCID: https://orcid.org/0000-0002-2069-0542

3 Hospital das Clínicas da Universidade Federal de Pernambuco - UFPE. Recife, PE - Brasil. ORCID: https://orcid.org/0000-0003-3538-5687

4 Departamento de Fisioterapia da Universidade Federal de Pernambuco - UFPE. Recife, PE - Brasil. ORCID: https://orcid.org/000-0002-3093-7481
}

\begin{abstract}
Resumo
Introdução: O paciente com Doença Renal Crônica (DRC) apresenta alterações respiratórias que persistem após o transplante e o restabelecimento da função renal. Objetivos: Avaliar a relação entre o tempo de transplante renal e sua influência sobre a força dos músculos respiratórios. Métodos: Foram avaliados voluntários adultos atendidos no Ambulatório de PósTransplante Renal do Hospital das Clínicas da Universidade Federal de Pernambuco, quanto à força muscular respiratória (manovacuometria), à função pulmonar (espirometria) e o nível de atividade física (Questionário Internacional de Atividade Física - IPAQ). Resultados: Foram avaliados 15 voluntários no total. No grupo $<154$ meses de transplante renal, 57,14\% dos voluntários apresentaram fraqueza muscular inspiratória (FMI), 57,14\% e $71,42 \%$ apresentaram, respectivamente, redução dos valores previstos de capacidade vital forçada (CVF\%) e do volume expiratório forçado no primeiro segundo $\left(\mathrm{VEF}_{1} \%\right.$ ), e $100 \%$ foram considerados ativos. No grupo $\geq 154$ meses de transplante renal, 50\% deles apresentaram FMI, 37,5\% mostraram valores de $C V F \%$ e $\mathrm{VEF}_{1} \%$ reduzidos e $62,5 \%$ foram classificados inativos. Todos os voluntários do estudo apresentaram fraqueza muscular expiratória. Conclusão: O grupo com menor tempo de transplante apresentou maior comprometimento da força muscular respiratória, sendo observado um nível de atividade física significativamente menor no grupo com maior tempo de realização do transplante. Não houve diferença em relação à função pulmonar entre os grupos.
\end{abstract}

Descritores: Transplante Renal. Músculos Respiratórios. Espirometria. Atividade física.
Abstract
Introduction: The chronic renal patient has respiratory changes that persist after transplantation, even after reestablishment of renal func- tion. Objectives: To evaluate the relationship between renal transplanta- tion time and its influence on the strength of the respiratory muscles. Methods: Adult volunteers were evaluated at the Outpatient Renal Transplant Clinic of the Hospital das Clínicas of the Federal University of Pernambuco, regarding respiratory muscle strength by means of manovacuometry, pulmonary function by spirometry and the level of physical activity by the International Activity Questionnaire Physics - IPAQ. Results: A total of 15 volunteers were evaluated. In the group $<154$ months of renal transplantation, $57.14 \%$ of the volunteers had inspiratory muscle weakness (IMF), 57.14\% presented reduced forced vital capacity (FVC) values, $71.42 \%$ had predicted expiratory volume forced in the first second reduced (FEV1\%) and $100 \%$ were considered active. In the group $\geq 154$ months of kidney transplantation, $50 \%$ had IMF, 37.5\% showed $\mathrm{FVC} \%$ and $\mathrm{FEV} 1 \%$ values reduced, and $62.5 \%$ were classified as inactive. All study volunteers had expiratory muscle weakness. Conclusion: The group with shorter transplant time presented greater impairment of 
respiratory muscle strength, and a significantly lower level of physical activity was observed in the group with longer transplantation time. There was no difference in lung function between groups.

Keywords: Renal transplantation. Respiratory Muscles. Spirometry. Physical activity.

\section{Introdução}

A Doença Renal Crônica (DRC) pode levar a complicações pulmonares por diferentes mecanismos: sobrecarga de líquido corporal no período interdialítico, infecções respiratórias, acidose metabólica, fibrose pulmonar, calcificações e alterações na relação ventilação/perfusão do pulmão ${ }^{1}$. Assim, o transplante renal é considerado a melhor opção de tratamento quando comparado à diálise de manutenção nesses doentes. Pacientes com DRC avançada possuem capacidade pulmonar reduzida, atribuída ao estado urêmico e ao descondicionamento imposto pela própria doença, não sendo claro o nível de recuperação da função pulmonar obtida pelo paciente pós-transplantado ${ }^{2,3}$.

Entretanto, as alterações encontradas no paciente com DRC persistem em pacientes transplantados, mesmo depois de restabelecida a função renal. O transplante renal, apesar de corrigir as complicações metabólicas da uremia, introduz outros problemas, como infecções pulmonares e fibrose, além de complicações não infecciosas, entre elas: atelectasias, derrame pleural e disfunção da musculatura diafragmática. Dessa forma, a diminuição de força muscular respiratória, piora da função pulmonar e redução da capacidade funcional são achados comuns nessa população ${ }^{4}$.

Apesar das alterações musculares e respiratórias dos pacientes transplantados estarem estabelecidas na literatura, não foram encontrados estudos que avaliassem se o tempo de transplante renal poderia influenciar nessas alterações. Portanto, é preciso caracterizar as modificações ocasionadas pelo transplante renal e assim implementar medidas terapêuticas mais eficazes e individualizadas para prevenir a incidência de tais mudanças. Nesse sentido, o objetivo principal deste estudo foi verificar a relação entre o tempo de transplante renal e sua influência na força muscular respiratória dos pacientes, e como objetivos secundários foram observar o comportamento da função pulmonar e o nível de atividade física.

\section{Materiais e métodos}

Esse estudo trata-se de uma série de casos realizado no Laboratório de Fisioterapia Cardiopulmonar do Departamento de Fisioterapia da Universidade Federal de Pernambuco (UFPE). A coleta de dados foi realizada após aprovação do Comitê de Ética em Pesquisa (CEP) do Centro de Ciências da Saúde da Universidade Federal de Pernambuco com número do parecer 2.003.778, entre os meses de abril a outubro de 2017.

A população foi constituída por voluntários adultos atendidos no Ambulatório de PósTransplante Renal do Hospital das Clínicas da Universidade Federal de Pernambuco (HCUFPE). A amostra foi obtida por acessibilidade e não probabilística.

Foram incluídos no estudo indivíduos com idade entre 18 e 59 anos, de ambos os sexos, com no mínimo 12 meses de transplante renal a partir da data de início da coleta, capazes de realizar os procedimentos avaliativos e responder aos questionários. Foram excluídos aqueles que apresentaram dificuldade de compreender os comandos verbais, de cooperação com os exames propostos, incapazes de realizar os testes de força muscular respiratória e/ou função pulmonar e tabagistas.

A triagem e a entrevista inicial dos voluntários foram realizadas no Ambulatório de PósTransplante Renal do HC-UFPE. Inicialmente, foi realizado o processo de divulgação e esclarecimento da pesquisa aos voluntários e, para aqueles que tiveram interesse em participar, foi solicitada a assinatura do Termo de Consentimento Livre e Esclarecido. Em seguida, foram agendadas as avaliações para co- 
leta de dados no Laboratório de Fisioterapia Cardiopulmonar (UFPE).

Os dados sociodemográficos, antropométricos, clínicos e laboratoriais foram coletados por meio de um questionário elaborado, sendo adquiridos diretamente do prontuário médico ou no momento da entrevista. Em seguida, o voluntário realizou um exame de função pulmonar por meio de espirometria e uma avaliação da força muscular respiratória por manovacuometria. Após esse momento, foi aplicado o Questionário Internacional de Atividade Física (IPAQ) na versão curta.

A espirometria foi realizada com o aparelho portátil (Micro Loop, Viasys ${ }^{\circledR}$ Health Care / England), de acordo com os padrões descritos pela American Thoracic Society 5 . Foram avaliados a capacidade vital forçada (CVF), volume expiratório forçado no primeiro segundo $\left(\mathrm{VEF}_{1}\right)$ e a relação $\mathrm{VEF}_{1} / \mathrm{CVF}$, sendo considerados os valores de referência para população brasileira adulta $^{6}$ e estabelecido como função pulmonar normal quando os valores previstos de CVF, $\mathrm{VEF}_{1}$ e $\mathrm{VEF}_{1} / \mathrm{CVF}$ foram iguais ou superiores a $80 \%$ do predito ${ }^{6}$.

A força muscular respiratória foi avaliada por meio da manovacuometria (MVD-300, Globalmed, Brasil) e realizada após 10 minutos de descanso seguido à espirometria, de acordo com os critérios da ATS/ERS 7 . Foram aferidas no mínimo cinco medidas, sendo registrado o maior valor obtido. Para análise de valores de normalidade das pressões respiratórias máximas foi utilizada a equação de normalidade proposta por Neder 8 .

Para determinar se o indivíduo apresenta fraqueza muscular respiratória foram considerados uma Pimáx inferior a $-60 \mathrm{cmH}_{2} \mathrm{O}$ para mulheres e $-80 \mathrm{cmH}_{2} \mathrm{O}$ para homens e Pemáx menor que $+120 \mathrm{cmH}_{2} \mathrm{O}$ para mulheres e +150 $\mathrm{cmH}_{2} \mathrm{O}$ para homens ${ }^{9}$.

O nível de atividade física foi identificado através do IPAQ e a análise dos resultados foi feita seguindo os critérios de frequência e duração; a população foi classificada em ativo, muito ativo, irregularmente ativo $\mathrm{A}$, irregularmente ativo B ou sedentários ${ }^{10}$. Para efeito de análise dos dados, foi considerada a classificação dos pacientes em "ativos" (categorias muito ativo e ativo) e "inativos" (categorias irregularmente ativo A e B e sedentário).

Os dados foram analisados através do programa estatístico Statistical Package for the Social Sciences (SPSS) for Windows versão 20.0. Para comparação dos dados basais entre os grupos foi utilizado o teste t-student. As distribuições de normalidade e de variância dos dados foram avaliadas, respectivamente, por meio do teste de Kolmogorov - Smirnov e de Levene, respectivamente. Os pacientes do estudo foram classificados de acordo com a mediana do tempo de transplante renal obtida nesse estudo (154 meses), sendo constituído um grupo $<154$ meses e outro grupo $\geq 154$ meses. Todas as análises e resultados dos pacientes foram apresentados a partir dessa classificação de tempo de transplante. Foi considerado como significativo um $\mathrm{p}$ valor $<0,05$.

\section{Resultados}

Foram abordados 122 voluntários para a pesquisa e após os critérios de elegibilidade foram excluídos 107 por recusarem participação na pesquisa, dificuldade de transporte que implica no comparecimento para os procedimentos de avaliação e outros motivos. Restaram 15 voluntários para a composição da amostra (Figura $1)$, sendo 10 homens.

As características antropométricas, clínicas, laboratoriais, nível de atividade física, função pulmonar e força muscular respiratória dos grupos $<154$ meses e $\geq 154$ meses encontram-se descritas na tabela 1 .

Considerando que os grupos foram semelhantes entre si para as variáveis acima mencionadas, exceto pelo tempo de transplante e nível de atividade física, as características individuais dos voluntários de cada grupo estão representadas nas tabelas 2 e 3 . Houve prevalência do sexo masculino em ambos os grupos, com média de 


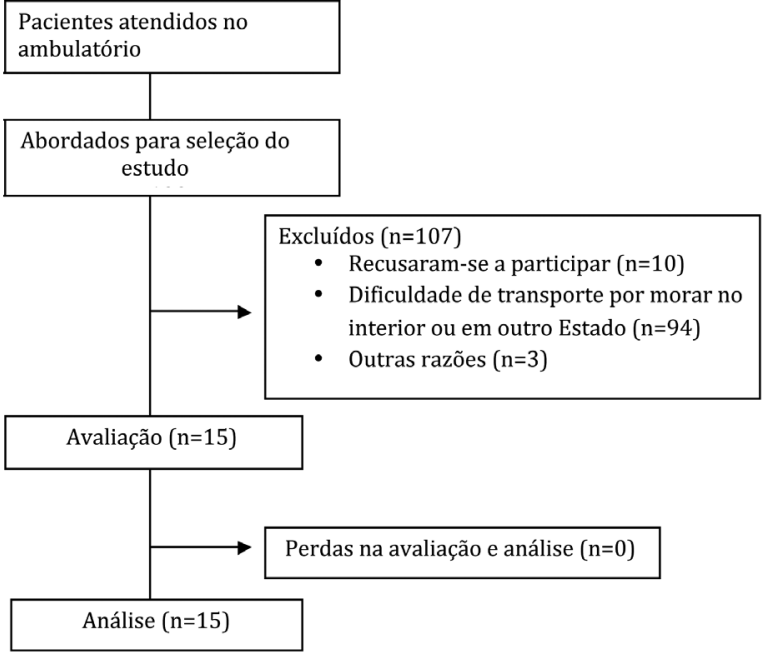

Figura 1: Fluxograma de constituição amostral

Fonte: Os autores.

Tabela l: Características clínicas, antropométricas, laboratoriais, nível de atividade física, dos pacientes do estudo de acordo com o tempo de transplante

\begin{tabular}{c|c|c|c}
\hline Variáveis & $\begin{array}{c}<154 \text { meses } \\
(\mathrm{n}=7)\end{array}$ & $\begin{array}{c}\geq 154 \text { meses } \\
(\mathrm{n}=8)\end{array}$ & \\
\hline & Média $\pm \mathrm{DP}$ & Média $\pm \mathrm{DP}$ & p-valor \\
\hline Idade (anos) & $38,86 \pm 12,32$ & $48,00 \pm 7,01$ & 0,116 \\
Tempo de TRS (meses) & $68,14 \pm 53,54$ & $90,75 \pm 31,85$ & 0,331 \\
Tempo de TX (meses) & $77,43 \pm 39,91$ & $195,63 \pm 37,44$ & 0,000 \\
IMC (kg/m²) & $25,33 \pm 5,12$ & $23,76 \pm 3,55$ & 0,499 \\
Cr (mg/dL) & $1,61 \pm 0,37$ & $1,82 \pm 0,76$ & 0,521 \\
\hline Sexo (M/F) & $\mathrm{n}(\%)$ & $\mathrm{n}(\%)$ & \\
\hline TRS (HD/DP) & $4(57,1) / 3(42,9)$ & $6(75)$ & \\
\hline Doença pulmonar prévia (S/N) & $2(28,6) / 5(71,4)$ & $2(25) / 6(75)$ & \\
Nível de atividade física (A/I) & $7(100)$ & $3(37,5) / 5(62,5)$ & \\
\hline
\end{tabular}

Legenda: $\mathrm{DP}=$ desvio padrão; $\mathrm{M}=$ masculino; $F=$ feminino; $I M C=$ Índice de massa corporal; $\mathrm{Tx}=$ Transplante; $\mathrm{TRS}=$ Terapia renal substitutiva; $\mathrm{HD}=$ Hemodiálise; $\mathrm{DP}=$ Diálise peritoneal; $\mathrm{Cr}=$ Creatinina; $\mathrm{S}=\operatorname{sim} ; \mathrm{N}=\mathrm{Não} ; \mathrm{A}=$ Ativo; I=Inativo.

Fonte: os autores.

idade $38,86 \pm 12,32$ anos para o grupo $<154$ meses e $48,00 \pm 7,01$ anos no grupo $\geq 154$ meses.

A tabela 2 apresenta as características do grupo $<154$ meses, no qual observamos que $57,14 \%$ dos voluntários apresentaram fraqueza muscular inspiratória e 100\% fraqueza muscular expiratória; $100 \%$ são considerados ativos pelo IPAQ. Além disso, foi observado que $57,14 \%$ e $71,42 \%$ apresentaram valores de $\mathrm{CVF}^{2}$ e e $\mathrm{VEF}_{1} \%$ reduzidos respectivamente, sendo sugestivo de alteração restritiva grau leve. O paciente $\mathrm{P} 4$ apresentou os menores valores espirométricos comparado ao restante do grupo e que coincidiu com o fato de passado de doença pulmonar (tuberculose). O paciente P7 apresentou os menores valores de Pimáx e Pemáx.

A tabela 3 apresenta as características do grupo $\geq 154$ meses, no qual observamos que $50 \%$ dos voluntários apresentaram fraqueza muscular inspiratória e 100\% deles apresentaram fraqueza muscular expiratória. Em relação aos parâmetros espirométricos, 37,5\% mostraram valores de $\mathrm{CVF} \%$ e $\mathrm{VEF}_{1} \%$ reduzidos. Foi observado que o paciente $\mathrm{P} 3$ apresentou menores valores e também coincidiu com o fato de passado de doença pulmonar (tuberculose). Houve prevalência de inativos pelo IPAQ $(62,5 \%)$ e o paciente P6 apresentou menores valores de Pimáx e Pemáx.

\section{Discussão}

Como resultados principais, o presente estudo observou que $57,14 \%$ dos indivíduos no grupo $<154$ meses e $50 \%$ do grupo acima desse tempo de transplante renal apresentaram fraqueza muscular inspiratória (FMI). Em relação à função pulmonar, foi observada redução da $\mathrm{CVF} \%$ e do VEF1\% respectivamente em $57,14 \%$ e $71,42 \%$ respectivamente nos indivíduos do grupo abaixo de 154 meses, e de 37,5\% para os indivíduos acima desse tempo de transplante para essas mesmas medidas. Com relação ao nível de atividade física, todos os indivíduos do grupo $<154$ 
Tabela 2: Características individuais dos pacientes transplantados renais do HC/UFPE com tempo de TX inferior a 154 meses

\begin{tabular}{|c|c|c|c|c|c|c|c|}
\hline Paciente & 1 & 2 & 3 & 4 & 5 & 6 & 7 \\
\hline Idade (anos) & 52 & 34 & 21 & 48 & 25 & 49 & 43 \\
\hline Sexo $(M / F)$ & $\mathrm{F}$ & M & M & M & M & $\mathrm{F}$ & $\mathrm{F}$ \\
\hline Tempo de TRS (meses) & 84 & 36 & 5 & 71 & 17 & 156 & 108 \\
\hline Tempo de TX (meses) & 113 & 95 & 13 & 100 & 29 & 108 & 84 \\
\hline $\mathrm{IMC}\left(\mathrm{Kg} / \mathrm{m}^{2}\right)$ & 33,50 & 23,90 & 26,80 & 24,80 & 18,90 & 29,43 & 20,00 \\
\hline $\mathrm{Cr}(\mathrm{mg} / \mathrm{dL})$ & 1,5 & 1,6 & 1,5 & 1,2 & 1,3 & 1,9 & 2,3 \\
\hline TRS (HD/DP) & $\mathrm{HD}$ & $H D$ & $\mathrm{HD}$ & $H D$ & $\mathrm{HD}$ & $\mathrm{HD}$ & $\mathrm{HD}$ \\
\hline Doença pulmonar prévia & SIM & NÃO & NÃO & SIM & NÃO & NÃO & NÃO \\
\hline Nível de atividade física (A/I) & A & A & A & A & A & A & A \\
\hline \multicolumn{8}{|l|}{ Espirometria } \\
\hline CVF \% do previsto & 77 & 82 & 76 & 60 & 84 & 98 & 67 \\
\hline $\mathrm{VEF}_{1} \%$ do previsto & 81 & 70 & 75 & 58 & 73 & 101 & 70 \\
\hline $\mathrm{VEF}_{1} / \mathrm{CVF}$ & 85 & 83 & 90 & 82 & 83 & 84 & 87 \\
\hline \multicolumn{8}{|l|}{ Força muscular respiratória } \\
\hline Pimáx obtido $\left(\mathrm{cmH}_{2} \mathrm{O}\right)$ & 59 & 59 & 87 & 54 & 132 & 108 & 54 \\
\hline Fraqueza inspiratória & SIM & SIM & NÃO & SIM & NÃO & NÃO & $\operatorname{SIM}$ \\
\hline Pemáx obtido $\left(\mathrm{cmH}_{2} \mathrm{O}\right)$ & 88 & 98 & 75 & 80 & 117 & 87 & 45 \\
\hline Fraqueza expiratória & SIM & SIM & SIM & SIM & SIM & SIM & SIM \\
\hline
\end{tabular}

Legenda: $\mathrm{M}=$ masculino; $\mathrm{F}=$ feminino; $\mathrm{TRS}=$ Terapia renal substitutiva; $\mathrm{Tx}=$ Transplante; $\mathrm{IMC}=$ Índice de massa corporal; $\mathrm{Cr}=$ Creatinina; $\mathrm{HD}=$ Hemodiálise; DP = Diálise peritoneal; $A=$ Ativo; I=Inativo; CVF = Capacidade vital forçada; $V E F_{1}=$ Volume expiratório forçado no primeiro segundo; Pimáx= Pressão inspiratória máxima; Pemáx = Pressão expiratória máxima.

Fonte: Os autores.

Tabela 3: Características dos pacientes transplantados renais do HC/UFPE com tempo de TX superior ou igual a 154 meses

\begin{tabular}{|c|c|c|c|c|c|c|c|c|}
\hline Paciente & 1 & 2 & 3 & 4 & 5 & 6 & 7 & 8 \\
\hline Idade (anos) & 59 & 43 & 44 & 56 & 48 & 45 & 51 & 38 \\
\hline Sexo (M/F) & $\mathrm{F}$ & M & M & M & M & $\mathrm{F}$ & M & M \\
\hline Tempo de TRS (meses) & 120 & 36 & 108 & 84 & 74 & 127 & 63 & 114 \\
\hline Tempo de TX (meses) & 200 & 162 & 180 & 180 & 269 & 228 & 154 & 192 \\
\hline $\mathrm{IMC}\left(\mathrm{kg} / \mathrm{m}^{2}\right)$ & 20,8 & 20,3 & 24,5 & 22,5 & 28,1 & 22,7 & 27,0 & 19,2 \\
\hline $\mathrm{Cr}(\mathrm{mg} / \mathrm{dL})$ & 1,0 & 1,8 & 1,8 & 1,5 & 1,3 & 1,3 & 3,3 & 2,6 \\
\hline TRS (HD/DP) & $\mathrm{HD}+\mathrm{DP}$ & $H D$ & $H D$ & $H D$ & $\mathrm{HD}+\mathrm{DP}$ & $H D$ & $\mathrm{HD}$ & $\mathrm{HD}+\mathrm{DP}$ \\
\hline Doença Pulmonar Prévia & NÃO & NÃO & SIM & NÃO & NÃO & SIM & NÃO & NÃO \\
\hline Nível de Atividade Física (A/l) & A & 1 & 1 & A & 1 & A & । & 1 \\
\hline \multicolumn{9}{|l|}{ Espirometria } \\
\hline CVF \% do previsto & 80 & 90 & 49 & 83 & 84 & 81 & 69 & 67 \\
\hline $\mathrm{VEF}_{1} \%$ do previsto & 81 & 92 & 58 & 90 & 90 & 69 & 66 & 92 \\
\hline$V_{E F} / C V F(\%)$ & 85 & 83 & 100 & 86 & 88 & 88 & 80 & 80 \\
\hline \multicolumn{9}{|l|}{ Força muscular respiratória } \\
\hline Pimáx obtido $\left(\mathrm{cmH}_{2} \mathrm{O}\right)$ & 69 & 101 & 79 & 93 & 58 & 38 & 122 & 61 \\
\hline Fraqueza inspiratória & NÃO & NÃO & SIM & NÃO & SIM & SIM & NÃO & SIM \\
\hline Pemáx obtido $\left(\mathrm{cmH}_{2} \mathrm{O}\right)$ & 63 & 106 & 92 & 91 & 108 & 41 & 108 & 98 \\
\hline Fraqueza expiratória & SIM & SIM & SIM & SIM & SIM & SIM & SIM & SIM \\
\hline
\end{tabular}

Legenda: $\mathrm{M}=$ masculino; F=feminino; TRS = Terapia renal substitutiva;

$\mathrm{Tx}=$ Transplante; IMC = Índice de massa corporal; $\mathrm{Cr}=$ Creatinina; $\mathrm{HD}=$ Hemodiálise; DP = Diálise peritoneal; $A=$ Ativo; I=Inativo; CVF = Capacidade vital forçada; $V F_{1}=$ Volume expiratório forçado no primeiro segundo; Pimáx= Pressão inspiratória máxima; Pemáx = Pressão expiratória máxima.

Fonte: Os autores. meses apresentaram-se ativos contra apenas $37,5 \%$ daqueles do grupo com maior tempo de transplante renal.

Verificamos que os voluntários de ambos os grupos eram predominantemente do sexo masculino, a HD foi o principal tipo de terapia renal substitutiva (TRS) antes do transplante, com tempo de TRS variando de 5 a 156 meses no grupo < 154 meses e variando de 36 a 127 meses no grupo $\geq 154$ meses. Nossos resultados corroboram com o perfil dos pacientes de outro estudo $^{11}$ que avaliou pacientes com DRC assistidos durante o pré-transplante renal, no qual a maioria era do sexo masculino (57,8\%), a HD foi o principal tipo de TRS utilizado no pré-transplante $(94,7 \%)$ e a maioria encontrava-se com mais de três anos de TRS.

Em relação à força muscular respiratória, em nossa amostra de voluntários, o grupo <154 meses apontou fraqueza muscular inspiratória. Os pacientes $\mathrm{P} 1, \mathrm{P} 2, \mathrm{P} 4$ e P7 encontram-se abaixo do limite inferior de normalidade para a Pimáx. No grupo $\geq 154$ meses $50 \%$ apresentaram fraqueza muscular inspiratória, onde os pacientes $\mathrm{P} 3, \mathrm{P} 5, \mathrm{P} 6$ e P8 não atingiram o valor de normalidade previsto para Pimáx.

Achados semelhantes foram observados no estudo de Jatobá et al ${ }^{12}$ que, ao avaliarem a força muscular respiratória em transplantados 
renais, verificaram comprometimento na capacidade muscular ventilatória, com diminuição de $38,2 \%$ da Pimáx em relação aos valores previstos. Cury, Brunetto e Aydos ${ }^{13}$ compararam a Pimáx e Pemáx de pacientes em hemodiálise e em pós-transplantados renais e sugeriram que, mesmo após o transplante, os pacientes não apresentaram a força muscular respiratória predita, corroborando com nossos achados.

Diferente do encontrado na nossa pesquisa, um estudo realizado por Guleria et $\mathrm{al}^{14}$ avaliou a função pulmonar e a força muscular respiratória uma semana antes, 30 dias e 90 dias após o transplante, concluindo que após o transplante renal houve uma melhora significativa na função pulmonar e aumento da força muscular respiratória. No entanto, esse estudo foi realizado com uma metodologia diferente por ter sido realizada avaliação dos parâmetros respiratórios imediatamente após o transplante renal.

Segundo Tavana e Mirzaei ${ }^{15}$, a duração mais longa da dependência de hemodiálise está associada à diminuição de força muscular respiratória, o que corrobora com a amostra do presente estudo, em que a maioria dos voluntários que apresentaram longo tempo de TRS desenvolveu fraqueza muscular inspiratória.

Outro resultado relevante em nosso estudo diz respeito à avaliação da função pulmonar e aos achados de valores abaixo do previsto para a $C V F$ e o $V_{E F}$. No entanto, a relação $V F_{1} / C V F$ apresentou-se normal. O paciente $\mathrm{P} 4$ do grupo < 154 meses e o paciente P3 do grupo $\geq 154$ meses apresentaram valores reduzidos e isso coincidiu com a história clínica pregressa de tuberculose pulmonar. É sabido que pacientes com tuberculose pulmonar, tratados ou não, podem apresentar sequelas e complicações pulmonares e extrapulmonares devido às alterações no calibre das vias aéreas e fibrose cicatricial, que reduz a capacidade pulmonar total, levando a padrões obstrutivos, restritivos ou mistos de comprometimento da função pulmonar ${ }^{16-17}$.

Os possíveis mecanismos propostos para explicar a diminuição da CVF com preservação da relação $\mathrm{VEF}_{1} / \mathrm{CVF}$ na maioria dos transplantados do estudo pode estar associado à presença de edema pulmonar crônico, de fibrose intersticial e calcificações do parênquima pulmonar e árvore brônquica, de infecções de repetição e de fibrose ${ }^{13}$.

Em relação ao nível de atividade física, todos os pacientes do grupo $<154$ meses foram considerados ativos e no grupo $\geq 154$ meses, $62,5 \%$ dos pacientes da amostra foram considerados inativos. Segundo Reboredo et $\mathrm{al}^{18}$, os pacientes em hemodiálise apresentam diminuição da capacidade funcional avaliada por meio do teste de caminhada de seis minutos, podendo ser atribuída à uremia, gerando baixa tolerância ao exercício. Sendo assim, após o transplante renal, os pacientes apresentam redução dos fatores que contribuem para a inatividade física, tais como o estado de uremia. Portanto, é esperado que tais pacientes apresentem melhora em seu desempenho físico e capacidade funcional, quando comparados ao período prétransplante ${ }^{19}$.

Galanti et $\mathrm{al}^{20}$ também sugeriram que após o transplante os pacientes renais retornaram às suas atividades de vida diária e se tornaram mais ativos após um programa de exercício; também verificaram que houve melhora na performance cardiovascular e na força muscular desses indivíduos, embora o tempo de realização de transplante de seu estudo tenha sido inferior ao nosso.

\section{Conclusão}

Na amostra estudada, 53,3\% dos pacientes transplantados renais apresentaram comprometimento da força muscular inspiratória independente do tempo de transplante, com fraqueza muscular expiratória presente em todos os voluntários. Também foi observada a redução nos valores preditos de $\mathrm{CVF} \%$ e $\mathrm{VEF}_{1} \%$ nos dois grupos sem diferença entre eles. Em relação ao nível de atividade, todos os indivíduos do grupo com maior tempo de transplante eram inativos. 


\section{Referências}

1. Kalender B, Erk M, Pekpak MA, Apaydin S, Ataman R, Serdengeçti K, et al. The effect of renal transplantation on pulmonary function. Nephron. 2002; 90(1): 72-77.

2. Rocha ER, Magalhaes SM, de Lima VP. Repercussion of physiotherapy intradialytic protocol for respiratory muscle function, grip strength and quality of life of patients with chronic renal diseases. J Bras Nefrol 2010; 32: 355-366.

3. Kovelis D, Pitta F, Probst VS, Peres CPA, Delfino VDA, Mocelin AJ et al. Função pulmonar e força muscular respiratória em pacientes com doença renal crônica submetidos à hemodiálise. J. Bras. Pneumol. 2008 Nov; 34(11): 907-912.

4. Ewert R, Opitz C, Wensel R, Dandel M, Mutze S, Reinke P: Abnormalities of pulmonary diffusion capacity in long-term survivors after kidney transplantation. Chest 2002; 122:639-644.

5. Lung function testing: selection of reference values and interpretative strategies. American Thoracic Society. Am Rev Respir Dis. 1991;144(5):1202-1218.

6. Pereira CAC, Sato T, Rodrigues SC. Novos valores de referência para espirometria forçada em brasileiros adultos de raça branca. J. Bras. Pneumol. 2007; 33(4): 397-406.

7. American Thoracic Society/European Respiratory Society ATS/ERS. Statement on respiratory muscle testing. Am J Respir Crit Care Med 2002; 166: 518624 .

8. Neder JA, Andreoni S, Lerario MC, Nery LE. Reference values for lung function tests. II. Maximal respiratory pressures and voluntary ventilation. Braz J Med Biol Res. 1999; 32(6): 719-727.

9. Caruso P, Albuquerque LP, Santana PV, Cardenas LZ, Ferreira JG, Prina E. Métodos diagnósticos para avaliação da força muscular inspiratória e expiratória. J Bras Pneumol, 2015; 41(2): 110-23.

10. Matsudo S, Araújo T, Matsudo V, Andrade D, Andrade E, Oliveira C, et al. Questionário Internacional de Atividade Física (IPAQ): estudo de validade e reprodutibilidade no Brasil. Rev Bras Ativ Fís Saúde. 2001; 6(2): 5-12.
11. Neto PML, Lima EVNCL. Avaliação da função pulmonar e qualidade de vida de pacientes renais crônicos em fase de pré-transplante renal. Rev Pesq Saúde. 2013; 14(3): 141-144.

12. Jatobá JPC, Amaro WF, Andrade APA, Cardoso FPF, Monteiro AMH, Oliveira MAM. Avaliação da função pulmonar, força muscular respiratória e teste de caminhada de seis minutos em pacientes portadores de doença renal crônica em hemodiálise. J Bras Nefrol 2008; 30:280-7.

13. Cury JL, Brunetto AF, Aydos RD: Efeitos negativos da insuficiência renal crônica sobre a função pulmonar e a capacidade funcional. Rev Bras Fisioter. 2010; 14 (2): 91-98.

14. Guleria S, Agarwal RK, Guleria R, Bhowmik D, Agarwal SK, Tiwari SC. The effect of renal transplantation on pulmonary function and respiratory muscle strength in patients with endstage renal disease. Transplant Proc. 2005; 37(2): 664-5.

15. Tavana S, Mirzaei S. The effect of renal transplantation on respiratory muscle strength in patients with end stage renal disease. Tanaffos. 2016; 15(2): 83-8.

16. Di Naso FC, Pereira JS, Schuh SJ, Unis G. Avaliação funcional em pacientes com sequela pulmonar de tuberculose. Rev Port Pneumol 2011; 17:216-21.

17. Chushkin MI, Ots ON. Comprometimento da função pulmonar após tratamento para tuberculose: o resultado final da doença? J. Bras. Pneumol. 2017; 43(1): 38-43.

18. Reboredo MM, Henrique DMN, Faria RS, Bergamini BC, Paula MGBeRB. Correlation between the distance covered in the six-minute walk test with peak oxygen uptake in end-stage renal disease patients on hemodialysis. Braz. J. Nephrol. (J. Bras. Nefrol.) 2007; 29(2): 85-89.

19. Ainsworth BE. How do I measure physical activity in my patients? Questionnaires and objective methods. British Journal of Sports Medicine 2009; 43:6-9.

20. Galanti G, Stefani L, Mascherini G, Petri C, Corsani I, Francini L et al. Short-term prospective study of prescribed physical activity in kidney transplant recipients. Intern Emerg Med 2016; 11: 61-67. 